\title{
A "circularisation" method to repair deformations and determine the centre of velocity map images
}

Jason R. Gascooke, Stephen T. Gibson, and Warren D. Lawrance

Citation: The Journal of Chemical Physics 147, 013924 (2017); doi: 10.1063/1.4981024

View online: http://dx.doi.org/10.1063/1.4981024

View Table of Contents: http://aip.scitation.org/toc/jcp/147/1

Published by the American Institute of Physics

\section{Articles you may be interested in}

Finite slice analysis (FINA)—A general reconstruction method for velocity mapped and time-sliced ion imaging

The Journal of Chemical Physics 147, 013913013913 (2017); 10.1063/1.4979305

Advantage of spatial map ion imaging in the study of large molecule photodissociation

The Journal of Chemical Physics 147, 013904013904 (2017); 10.1063/1.4975671

Velocity map imaging of ions and electrons using electrostatic lenses: Application in photoelectron and photofragment ion imaging of molecular oxygen

The Journal of Chemical Physics 68, (1998); 10.1063/1.1148310

Nonadiabatic laser-induced alignment of molecules: Reconstructing

$\# \& \# x 1 d 5 b c ; \& \# x 1 d 5 c 8 ; \& \# x 1 d 5 c c ; \& \# x 1 d 7 e 4 ; \& \# x 1 d 73 d ; \#$ directly from

\#\&\#x1d5bc;\&\#x1d5c8;\&\#x1d5cc;\&\#x1d7e4;\&\#x1d73d;2D\# by Fourier analysis

The Journal of Chemical Physics 147, 013905013905 (2017); 10.1063/1.4975817

Perspective: $\mathrm{C} 60+$ and laboratory spectroscopy related to diffuse interstellar bands

The Journal of Chemical Physics 146, 160901160901 (2017); 10.1063/1.4980119

Ion-ion coincidence imaging at high event rate using an in-vacuum pixel detector

The Journal of Chemical Physics 147, 013919013919 (2017); 10.1063/1.4981126

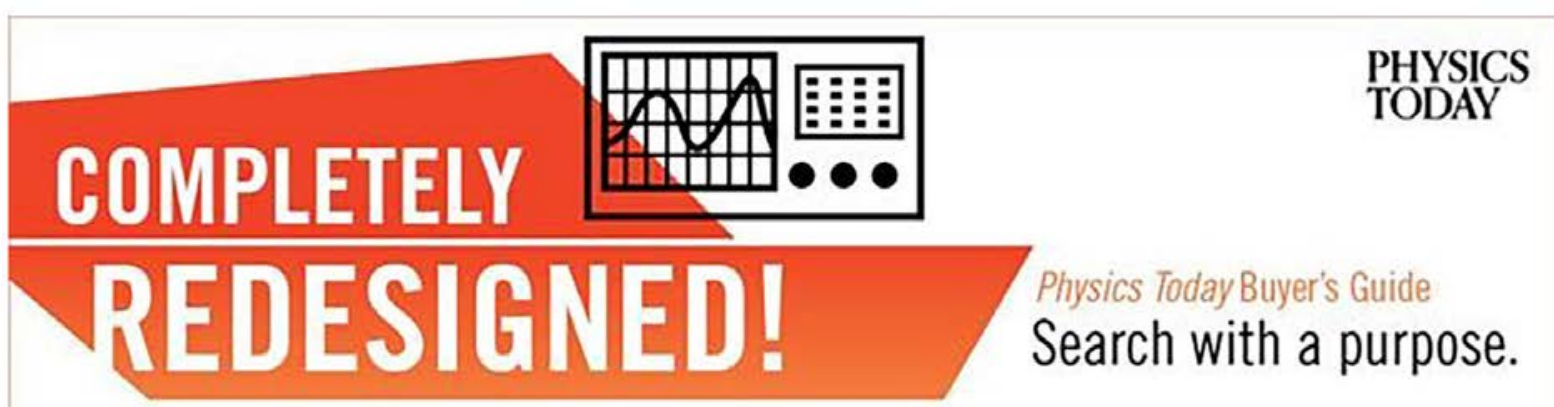




\title{
A "circularisation" method to repair deformations and determine the centre of velocity map images
}

\author{
Jason R. Gascooke, ${ }^{1}$ Stephen T. Gibson, ${ }^{2}$ and Warren D. Lawrance ${ }^{1, a)}$ \\ ${ }^{1}$ School of Chemical and Physical Sciences, Flinders University, GPO Box 2100, Adelaide, \\ South Australia 5001, Australia \\ ${ }^{2}$ Research School of Physics and Engineering, The Australian National University, Canberra, \\ ACT 2601, Australia
}

(Received 8 February 2017; accepted 4 April 2017; published online 1 May 2017)

\begin{abstract}
A problem besetting the analysis of velocity map images, particularly those of photoelectrons, is the presence of distortions that cause the features in the image to deviate from circularity, leading to a loss of resolution in the spectrum extracted. A method is presented to repair such distortions based on fitting the angular behaviour of each of the ring structures to a trigonometric expansion. The repair function allows the intensity at any value of radius and angle to be mapped to a new position that removes the distortion and returns the features to circular. While the method relies on the analysis of the structure in an image, it could also be applied to determine the "repair function" using a calibration image (or series of images) for the experiment. Once the image has been circularised it can be processed by any of the approaches that have been developed for that purpose. The analysis also enables the image centre to be determined with high accuracy. The fitting method utilises an inverse Abel transformation of the image in polar coordinates as a means to reshape the image into a series of spectral features in order to determine the radial position of features at each angle. Although the velocity distribution is not in general spherically symmetric and so this is not a mathematically correct means to extract the velocity distribution, the feature positions are accurately reproduced in the resulting spectrum while the intensity and anisotropy parameters can be remarkably close to those obtained using the proper inverse Abel transformation of the image. Published by AIP Publishing. [http://dx.doi.org/10.1063/1.4981024]
\end{abstract}

\section{INTRODUCTION}

Since the introduction of the ion imaging technique by Chandler and Houston 30 years ago, ${ }^{1}$ it has become a standard part of the experimental toolkit for molecular dynamics studies. The introduction of velocity mapping, the so-called velocity map imaging (VMI) technique, a decade later significantly improved the resolution attainable. ${ }^{2}$ Although initially applied to imaging ions, the technique has also been used to image electrons and has become a powerful technique in photoelectron spectroscopy of neutrals and ions. ${ }^{3-8}$

During conventional VMI experiments, ionised photoreaction products or photoelectrons are produced in a small volume by a short laser pulse and accelerated toward a detector using electric fields that place the particle on the detector at a position governed by its initial velocity. As the ions/electrons travel toward the detector they expand spherically as determined by the velocity they gained during the photodissociation (ions) or ionisation (electrons) event, and it is the two dimensional (2D) projections of these Newton spheres that are measured when the charged particles hit the detector. Assuming cylindrical symmetry about the laser polarization, a slice of the original 3D particle distribution has traditionally been obtained using an inverse Abel transformation, although

\footnotetext{
a) Author to whom correspondence should be addressed. Electronic mail: warren.lawrance@flinders.edu.au.
}

alternative methods have been developed. An early comparison of the various approaches taken was provided in Ref. 9. New approaches continue to be developed, ${ }^{10,11}$ with a more recent summary of the various techniques given in Ref. 11. Alternatively, the 3D particle distribution can be measured directly with the slice imaging experimental technique, ${ }^{12,13}$ although this is not appropriate for electron detection due to the short flight times involved.

Ideally, the particles' spherical velocity distribution gives rise to perfect circles in the experimental image; however, in practice experimental images may deviate from circularity due to, for example, stray magnetic fields or optical distortion of the camera lens that images the particle detector. The difficulty of completely eliminating stray magnetic fields coupled with the low electron mass makes photoelectron images particularly sensitive to distortion. The effect of distortion is to reduce the radial (and hence velocity and kinetic energy) resolution, since a particular energy peak will "walk" in radial position, depending on the angular position of the detector. This leads to a loss of spectral resolution. To minimise the effect, the analysis is often confined to a limited section of the image. For example, in their original velocity map imaging paper, Eppink and Parker ${ }^{2}$ addressed this problem by only integrating over a single quadrant. Alternatively, imposing a circular distribution of particles improves the kinetic energy resolution at the expense of an uncertainty in the absolute kinetic-energy position of the transition. Eppink, Wu, and Whitaker have considered the 
effects of simple distortions when comparing reconstruction methods. ${ }^{9}$

To illustrate the issue, Fig. 1(a) shows a photodetachment VMI image of $\mathrm{O}^{-}$obtained in The Australian National University (ANU) laboratory. While not immediately obvious to the naked eye, the rings are not exactly circular. We have included a circle on the image (dashed lines in red) to guide the eye and make the presence of deformations more apparent. The spectral information is extracted by taking an inverse Abel transform of the image to produce a slice through the centre of the expanding spheres and integrating over the spherical angles to reduce the intensity to a function of $r$ only. ${ }^{3,11}$ Since the image is not perfectly circular, the peaks in the angular integrated spectrum have a width larger than arises from the width of the rings in the image, resulting in a loss of spectral resolution. Fig. 1(b) shows the inverse Abel image ${ }^{14}$ of Fig. 1(a) and Fig. 1(c) shows the spectrum extracted by integration of the complete image. In contrast, Fig. 1(d) shows the spectrum obtained by integrating over a small $\left(6^{\circ}\right)$ wedge. This wedge angle is too small for deformations to affect the resolution and thus shows the resolution achievable by the instrument. The significant reduction in the resolution from the features in Fig. 1(d) to Fig. 1(c) when using the full image is apparent.

While narrowing the integration to small angular sections can improve the resolution, it is not an altogether satisfactory solution since this approach discards much of the data, lowering the signal to noise ratio for a given number of laser shots and does not provide the total cross section. Only when an appropriate quarter image is used (i.e., one axis is parallel to the laser polarisation direction) will the photoelectron distributions calculated from angular segments give the correct intensities because of anisotropy in the angular distributions. When integrating over large angular segments such as a quadrant, closely spaced rings in the image can become merged. A better approach would be to "repair" such distortions, returning the image to circular prior to the analysis. By using a complete image there is a redundancy since the four quarters should be identical, which provides a check for the internal consistency of the results. The use of the entire image compared to a single quadrant gives an improvement in the signal of a factor of 4 and the signal to noise ratio, which is proportional to the square root of the number of counts, a factor of 2, potentially reducing collection times.

Various methods to overcome distortion have been reported. Gascooke ${ }^{15}$ introduced a radial repair method, whereby the image was broken up into wedges of equal angles, akin to cutting a pie. One wedge was taken as the reference, with the remaining wedges being stretched or compressed, with appropriate intensity scaling such that the structure in the wedges had the same radial dependence as the reference wedge. Ryazanov has recently reported a similar scheme. ${ }^{16}$ The method reported by Gascooke has been incorporated as a part of the data analysis for the high resolution photoelectron spectrometer at the Australian National University $8,17,18$ and in various publications from the Flinders group. ${ }^{19-21}$ The approach has recently been improved to include a trigonometric fitting of the angular distortion, as discussed briefly in a recent publication. ${ }^{21}$ Basis function inverse Abel (a)

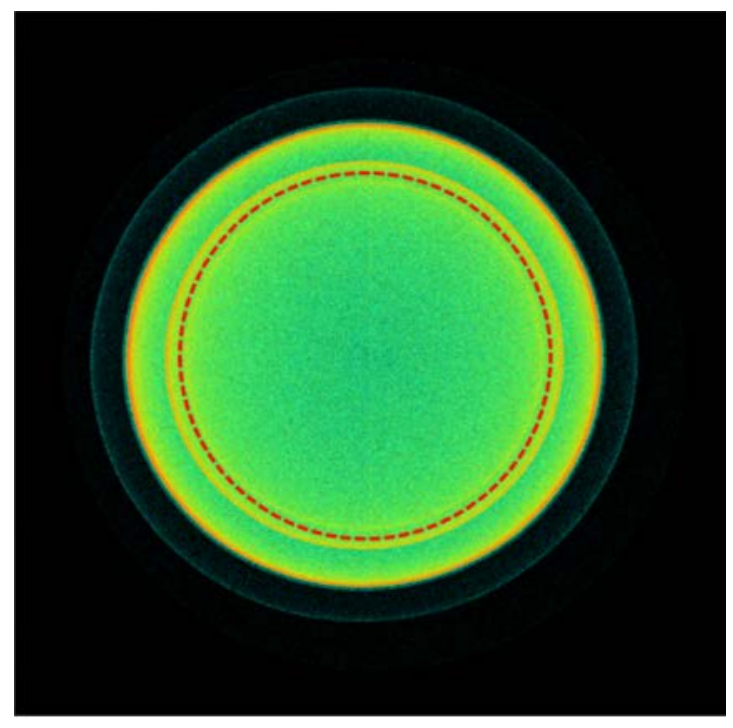

(b)

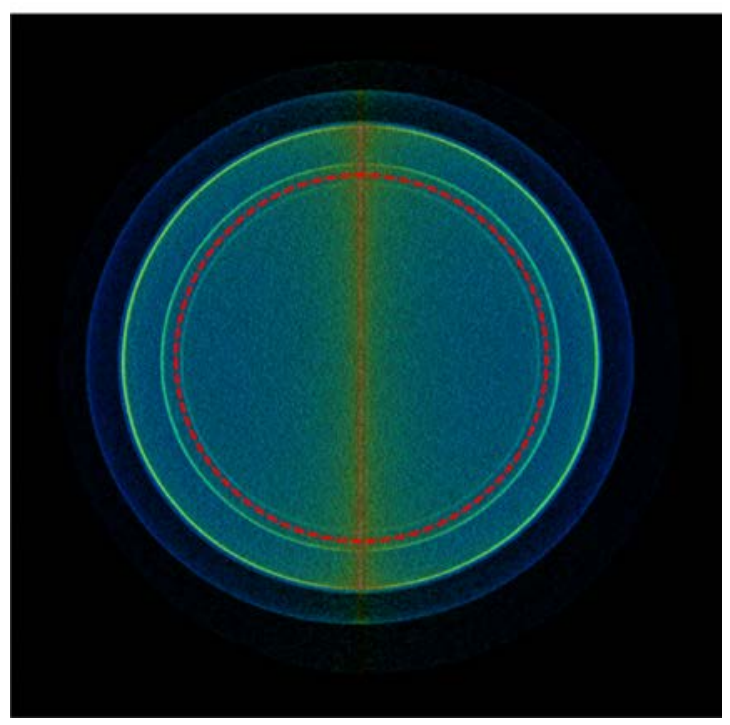

(c) 70
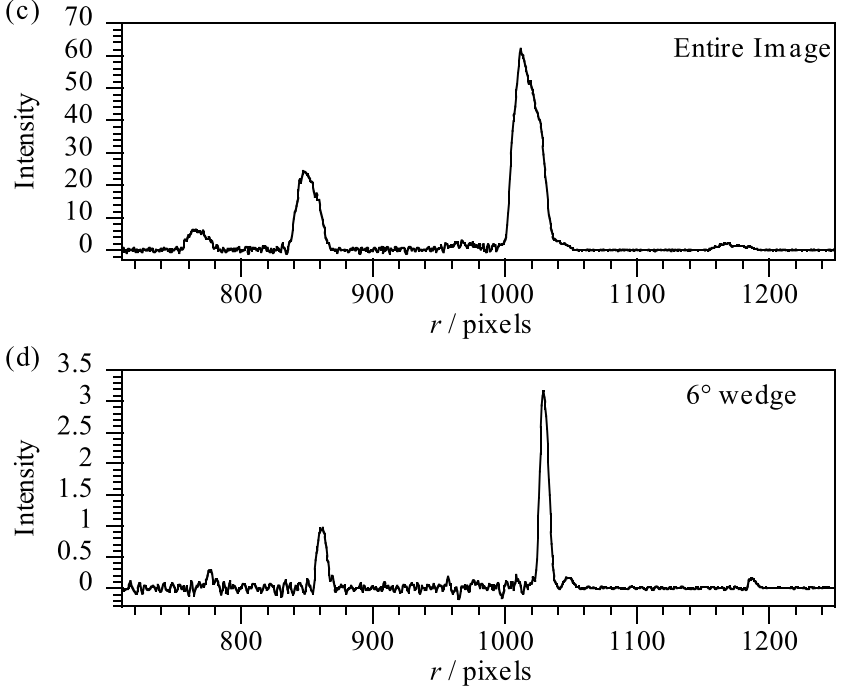

FIG. 1. (a) A VMI image of the electrons generated by photodetachment of $\mathrm{O}^{-}$at $812.51 \mathrm{~nm} .{ }^{17}$ The dashed red circle on the image is provided to guide the eye and make the presence of deformations more discernible. (b) The inverse Abel transform of this image. (c) The "spectrum" (plotted versus radius) extracted from the inverse Abel transformed image shown in (b). (d) A plot of the intensity versus radius for a $6^{\circ}$ wedge $\left(\theta=202^{\circ}-208^{\circ}\right)$ of the inverse Abel transformed image shown in (b). Comparing (c) and (d) shows the loss of resolution due to non-circularity of the image. 
transform methods take a different approach and fit circularly symmetric functions to the image, effectively averaging the distortion..$^{9,22,23}$

Recently, we have been developing an improved method for repairing distorted images, building on the method reported by Gascooke. ${ }^{15}$ The purpose of the present paper is to present this method, which we refer to as "circularisation." The method also provides a means to determine the centre of the image with quite high precision. Several authors have commented on the need for accurate determination of the image centre ${ }^{22,23}$ and a variety of methods have been used. ${ }^{22-24}$ The circularisation technique is demonstrated using photoelectron images measured in the Flinders and ANU laboratories. Once circularised, the image can be processed using any of the methods available for that purpose.

\section{REMOVING DISTORTION FROM IMAGES}

\section{A. Overview of the technique}

Structured VMI images generally consist of a number of concentric rings. In essence, the circularisation method identifies a functional form for the image distortions by fitting the angular behaviour of each of the rings to a trigonometric expansion. Doing this for a number of features of different radii allows the angular variation at other radii to be determined by interpolation. The result is a function that allows the intensity at any value of radius and angle to be remapped to remove the distortion and return the features to circular. The process does not alter the intensity/counts within each ring.

As apparent in Fig. 1(a), it can be difficult to see the distortions simply by viewing the image. We have found that they are much more apparent after converting the VMI image to polar coordinates (radius $r$ and angle $\theta$ ) and plotting it as a $(r, \theta)$ plot with $r$ along the horizontal axis and $\theta$ along the vertical axis. We refer to these as polar coordinate (PC) images. A PC image of the VMI image shown in Fig. 1(a) is shown in Fig. 2(a). In this and subsequent images, $\theta$ is defined as the anticlockwise trigonometric angle from the $x$ (horizontal) axis. (Experimentally, $\theta$ is often used to denote the angle from the laser polarisation for the purpose of defining the anisotropy parameter, $\beta$, and hence is measured from the horizontal or vertical axis depending on the experimental configuration.) In the absence of deformations and using the correct image centre (see below), the PC image would show a series of straight lines parallel to the $\theta$ axis (i.e., vertical lines), one for each of the rings seen in the image. The deformations in the VMI image are apparent in the PC image, with "wiggly" features being observed instead of straight lines. The circularisation process determines the function describing these wiggles and then uses it to repair the image.

It is important to note that if the value for the image centre is incorrect, the PC image will yield a "wiggly" line rather than a straight one even for a perfectly circular image. This artifact provides a method for determining the position of the centre, as discussed in Section II B, step 4, with further details provided in the supplementary material. Figs. 1(b)-1(d) have been generated using the correct centre as determined by the circularisation procedure.
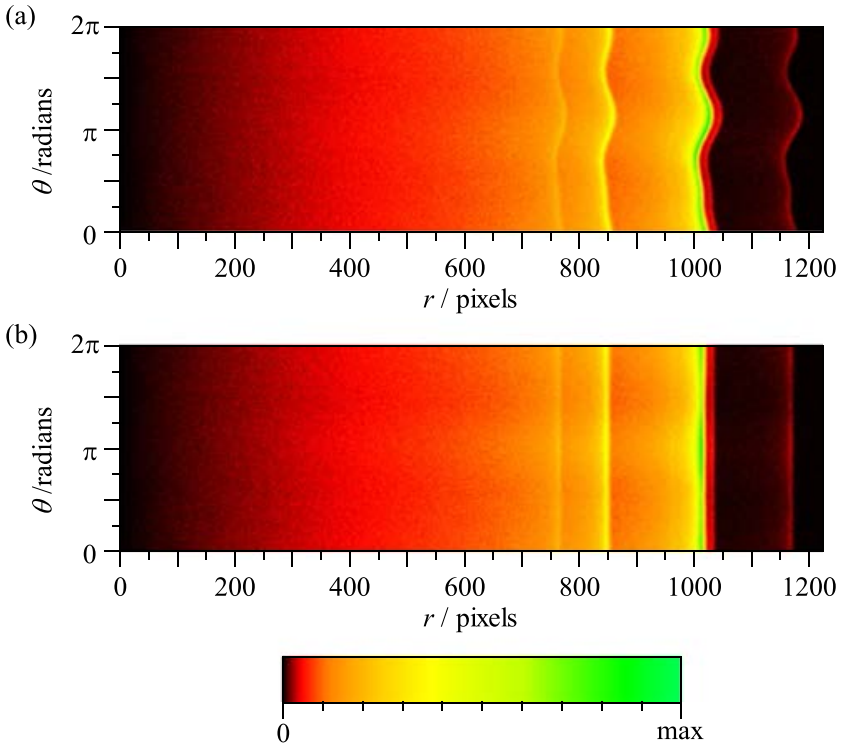

FIG. 2. (a) A polar coordinate (PC) image of the VMI image shown in Fig. 1(a). The deformations in the VMI image are revealed by the "wiggly" nature of the lines observed. (b) The PC image for the circularised VMI image showing that the vertical lines have been straightened by the circularisation process. This illustrates the excellent circularity of the repaired image.

\section{B. The technique step by step}

It is most instructive to describe the method by working through an example. An example based on a synthetic image has been chosen since the parameters used to generate the distortion are known: the distortion parameters determined during the analysis can thus be compared with them and the repaired image compared with the undistorted original, providing a test of the method. The undistorted synthetic VMI image is shown in Fig. 3(a) and the distorted version in Fig. 3(b). The synthetic image is constructed as 5 Gaussian line shapes whose widths were varied to mimic the behaviour of VMI experiments. The radii of these features and their full width at half maximum (FWHM) are, in units of pixels, 50 (4.71), 100 (2.71), 150 (1.57), 200 (1.18), and 250 (1.18). The features are centred at pixel location $(301,301)$. The intensity variation with the angle has been set using the usual $\beta$ parameter form ${ }^{25}$

$$
I(\phi)=I_{0}\left(1+\frac{1}{2} \beta\left(3 \cos ^{2} \phi-1\right)\right)
$$

with $\beta=0.67$ used for the synthetic image. Here, $\phi$ is the angle from the laser polarisation, which is assumed to be vertical, i.e., $\phi=\pi / 2-\theta$. The synthesized distortion is represented by a function of the form

$$
r_{d}(\theta)=r_{u}\left(1+\sum_{n=1}^{N}\left(a_{n} \sin n \theta+b_{n} \cos n \theta\right),\right.
$$

where $r_{d}$ is the distorted radius (i.e., the radius observed experimentally), $r_{u}$ is the undistorted (true) radius, $N$ is the number of terms in the expansion used to describe the distortion, and $a_{n}$ and $b_{n}$ are constants that describe its magnitude. Table I lists the values used for these parameters. This distortion changes linearly with radius, which is the dominant effect expected experimentally since the distortion arises from misshapen expanding charged particle spheres whose radii are 


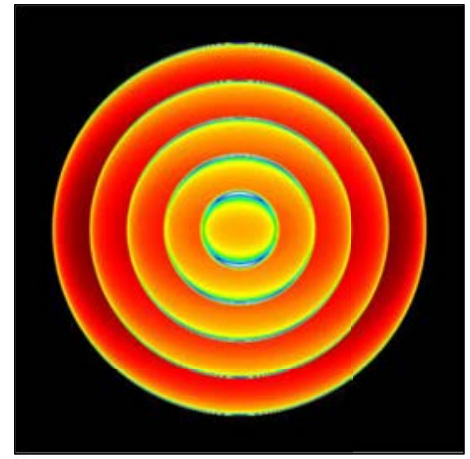

(b)

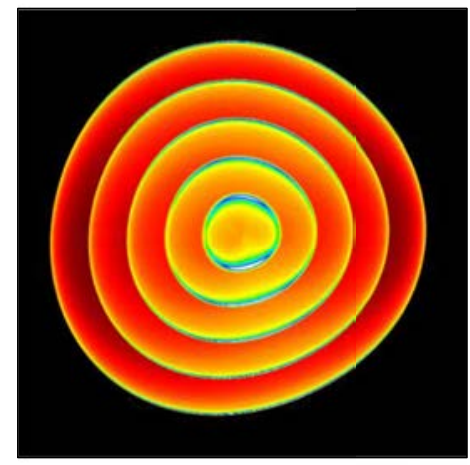

(c)

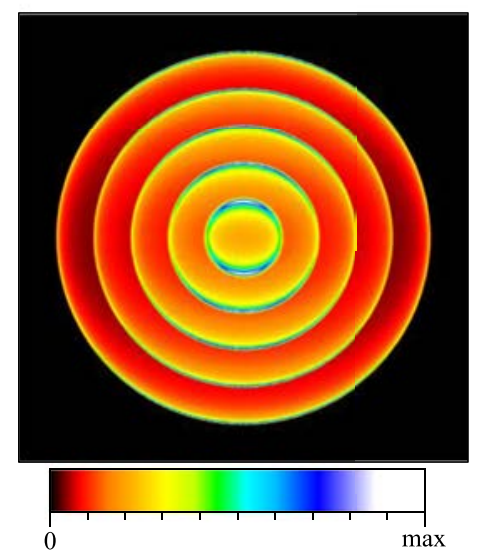

(d)

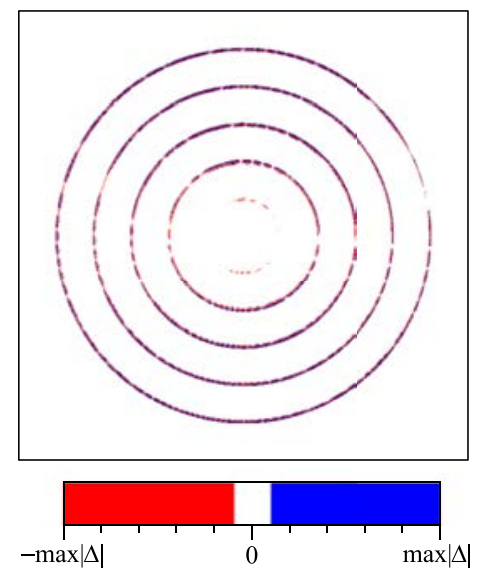

FIG. 3. (a) The synthetic VMI image used in the worked example describing the circularisation method. (b) The image after it has been distorted using a trigonometric function as described in the text. The parameters used for the function are shown in Table I. (c) The final repaired, circularised image. (d) A difference image showing the original minus the circularised image. The difference is constant in $r$ which is only possible if the image is properly circularised. There are some minor differences apparent and these are due to the pixelated nature of the image leading to a slight broadening of the sharp features.
TABLE I. The constants $a_{n}$ and $b_{n}$ involved in generating the distorted VMI image used in the worked example describing the circularisation method.

\begin{tabular}{lrrrrr}
\hline \hline & \multicolumn{2}{c}{ Input } & & \multicolumn{2}{c}{ Fitted $^{\mathrm{a}}$} \\
\cline { 2 - 3 } \cline { 5 - 6 }$n$ & \multicolumn{1}{c}{$a_{n}$} & $b_{n}$ & & \multicolumn{1}{c}{$a_{n}$} & \multicolumn{1}{c}{$b_{n}$} \\
\hline 1 & $2.00 \times 10^{-2}$ & $-4.00 \times 10^{-2}$ & & $2.01 \times 10^{-2}$ & $-4.01 \times 10^{-2}$ \\
2 & $1.80 \times 10^{-2}$ & $4.00 \times 10^{-3}$ & & $1.80 \times 10^{-2}$ & $4.02 \times 10^{-3}$ \\
3 & $-1.20 \times 10^{-2}$ & $1.80 \times 10^{-2}$ & & $-1.20 \times 10^{-2}$ & $1.79 \times 10^{-2}$ \\
4 & $6.00 \times 10^{-4}$ & $-2.00 \times 10^{-4}$ & & $6.10 \times 10^{-4}$ & $-1.83 \times 10^{-4}$ \\
\hline
\end{tabular}

${ }^{\text {a The one standard deviation values from the least squares fit of the parameters are in the }}$ range 4-6 $6 \times 10^{-6}$.

determined by the particles' velocities. Where present, nonlinear radial effects can be accounted for once the image has been circularised. In that case, the energies of the features must be known so that the equation for the non-linear radial repair can be determined (see Section III A for an example). No noise term is included in the synthetic image.

The steps involved in the analysis are as follows:

1. Initial centre determination. The process utilises PC images whose generation requires a value for the image centre. The centre position is checked and adjusted during the analysis (step 4), and a reasonable initial estimate suffices. In this example, we have simply averaged the values for the left-most and right-most positions of the smallest ring (small rings are least affected by distortions) and the top-most and bottom-most positions to give the $x$ and $y$ values, respectively. This gives a centre pixel location of $(302.5,300.0)$, which compares with the true value of (301, 301).

2. Generate the PC image and its inverse Abel transform. A PC image is generated by converting the intensity at $(x, y)$, $I(x, y)$, into intensity at $(r, \theta), I(r, \theta)$, based on the distance $r$ from the chosen centre, $r=\sqrt{\left(x-x_{c}\right)^{2}+\left(y-y_{c}\right)^{2}}$, and angle $\theta$ (defined above). The PC image generated from Fig. 3(b) is shown in Fig. 4(a). The aim is to analyse the features in the PC image to determine their angular behaviour. There are a number of ways this could be done, for example, using edge finding routines or simply searching for the maximum value within a region. We process the PC image using an inverse Abel transform as a means to reshape the image into a series of spectral features. Each horizontal line, i.e., a constant $\theta$ value, is transformed by the inverse Abel transform. We find that although the velocity distribution is not in general spherically symmetric, i.e., $\beta \neq 0$ in general, and thus this is not a mathematically correct means to extract a velocity distribution, the resulting spectrum and anisotropy parameters can be remarkably close to those obtained using the proper inverse Abel transformation of the image (see supplementary material). Importantly, the positions of the features are accurately determined in the inverse Abel transform of the PC image, which consists of a series of Gaussian or Gaussian-like features whose position in $r$ varies with $\theta$. As the arcs being integrated over get longer with increasing $r$ for a given $\delta \theta$, the intensity must be scaled by dividing by $r$ to normalise for this before performing the inverse Abel 

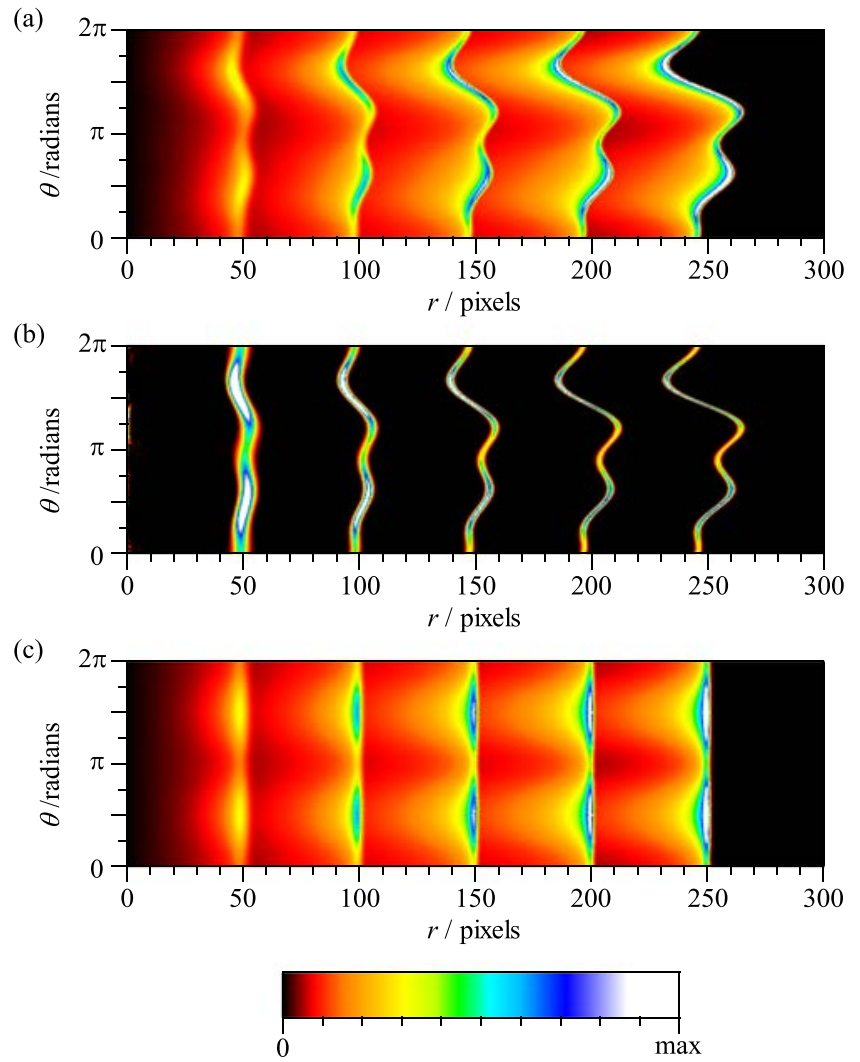

FIG. 4. (a) The PC image generated from Fig. 3(b) using $\theta$ bins of $2^{\circ}$ and $r$ bins of 0.5 in units of the pixel size in the original $(x, y)$ image. (b) The inverse Abel transformed PC image. The intensity in the PC image was divided by $r$ prior to applying the inverse Abel transformation (see text). (c) As for (a) but for the circularised (repaired) VMI image. Note the straight lines for the circularised image.

transformation. The inverse Abel transformed PC image is shown in Fig. 4(b). The inverse Abel transformation of the PC image leads to noise near $r=0$ but, provided that there is no structure at very low radii, this causes less of a problem than in the case of the inverse Abel transform of the VMI image. Taking the inverse Abel transform of the original image introduces a noise strip down the centre of the image, resulting in regions of the PC image around $\theta=90^{\circ}$ and $270^{\circ}$ that would need to be masked out in the remaining analysis (see Fig. 1(b) as an example).

3. Fit a trigonometric series to each "line" in the inverse Abel PC images. A trigonometric series is used to account for the angular behaviour of each of the features shown in Fig. 4(b). Each feature is first extracted from the full image as a separate image over the appropriate radial segment, and then fitted independent of the other features. The features are assumed to be Gaussian in $r$ and have an intensity variation in $\theta$ that is described by the usual $\beta$ parameter (Eq. (1)), ${ }^{25}$ although for experiments where this is inappropriate other functions could be used to describe the intensity variation with the angle. The function used to fit the $i$ th feature is

$$
I(r, \theta)=I(\phi) \exp \left(-\frac{\left(r-r_{g}\right)^{2}}{2 \sigma_{i}^{2}}\right)+c
$$

where $r_{g}(\theta)=r_{i}+\sum_{n=1}^{N}\left(A_{n}^{(i)} \sin n \theta+B_{n}^{(i)} \cos n \theta\right)$ is the position of the Gaussian and $I(\phi)=I_{0}\left(1+\frac{1}{2} \beta_{i}\left(3 \cos ^{2}(\phi)-1\right)\right)$, with $\phi=\pi / 2-\theta$ (see Eq. (1)), is its intensity. $r_{i}$ is the unperturbed radius of the feature, while $A_{n}^{(i)}$ and $B_{n}^{(i)}$ are the trigonometric coefficients describing its distortion. $c$ is a constant to account for any intensity offset should the background be non-zero. $\sigma_{i}$ is the standard deviation of the Gaussian, which is related to its full width at half maximum by FWHM $=2 \sqrt{2 \ln 2} \sigma_{i}$. The number of terms, $N$, required in the trigonometric series depends on the deformation and could be assessed in a number of ways. We determine $N$ by comparing the size of the term with its uncertainty since a large uncertainty indicates that the term is ill-determined and not required. The fit returns values of $r_{i}$ and $\beta_{i}$ and a set of $A_{n}^{(i)}$ and $B_{n}^{(i)}$ values whose number depends on the value of $N$ required.

In principle, if the analysis has been performed using the correct centre (see step 4 below) and involves every spectral feature in the image, much of the information to be extracted from the image is known at this point and a reconstruction of the circularised image (steps 5 and 6) may not be necessary. The anisotropy parameter $\beta$ will not necessarily be properly determined because the inverse Abel transform was performed on the PC image rather than the VMI image, although the differences can be small (see supplementary material). However, weak features can be difficult to include in the circularisation analysis but emerge when the image is repaired and processed to yield the spectrum.

4. Check for the correct image centre. An incorrect choice of image centre is manifested in $A_{1} \sin \theta$ and $B_{1} \cos \theta$ terms (see supplementary material) but such terms can also arise from the deformation. At this point, it is necessary to assess whether the image centre being used is correct or requires a change. If the centre needs changing, steps 2 and 3 are repeated with the new centre value so that the constants reflect only the deformation. To assess if the centre is correct, the $A_{1}$ and $B_{1}$ values are plotted as a function of $r$. Since at $r=0$ the angular deformation vanishes, extrapolating these plots to $r=0$ gives the $A_{1}$ and $B_{1}$ values arising from the incorrect choice of centre, and hence the correction required (see supplementary material). The function is linear if the deformation is purely radial, which is expected, but non-linear functions may be needed if this is not the case. In our experience, a linear function has been adequate. In the example at hand, the plot indicates that the chosen centre is incorrect and that the corrected value is $(301.03,300.98)$ pixels, which is reassuringly close to the exact value of (301.00, 301.00). With a maximum image radius of 250 pixels, this represents an error in the centre of $\sim 0.01 \%$. Steps 2 and 3 were repeated using the new centre position. The $r$ dependence of the new $A_{1}$ and $B_{1}$ terms showed that the centre had been determined with sufficient accuracy, as expected.

5. Determine the radial dependence of the angular deformation terms, $A_{n}$ and $B_{n}$. When using the correct centre, 


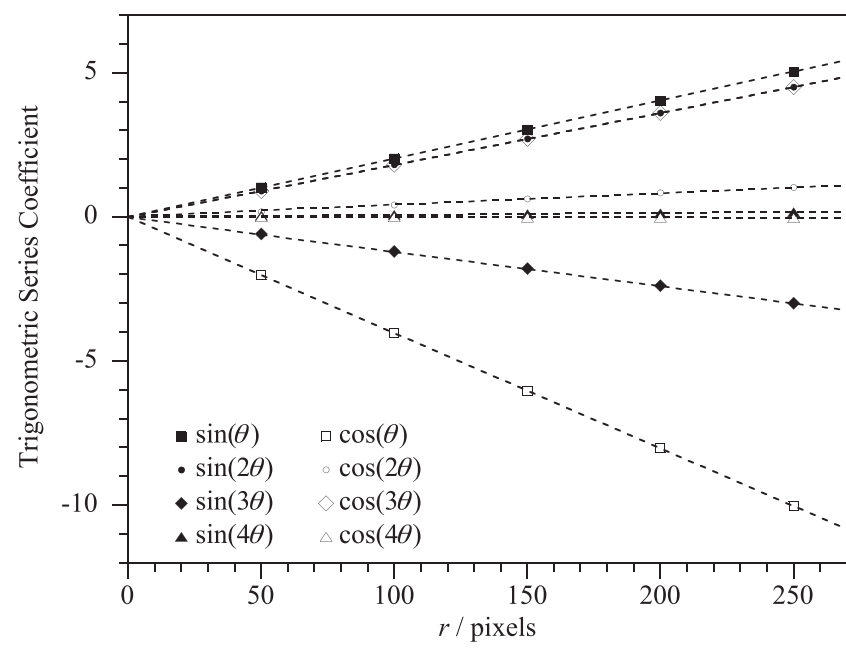

FIG. 5. A plot of the $A_{n}\left(r_{i}\right)$ and $B_{n}\left(r_{i}\right)$ values as a function of $r$ and the straight line fits. Based on the use of an angular distortion to generate the test image, the plots should be linear and pass through zero. The slopes of the plots should match the values used to deform the test image; the values are compared in Table I.

step 3 produces a set of $A_{n}^{(i)}$ and $B_{n}^{(i)}$ for particular $r$ values, $r_{i}$. The $r$ dependence of these constants is determined by plotting these points as functions of $r$ and fitting the values to a function. A linear function was used to generate the deformation in our synthetic image and so the plots in this case should be linear and pass through zero, i.e., $A_{n}(r)=a_{n} r$ and $B_{n}(r)=b_{n} r$, where $a_{n}$ and $b_{n}$ are the slopes of the plots. This is the case shown in Fig. 5. The parameters extracted should match those used to generate the deformation. A comparision between the two sets (Table I) shows that the circularisation process has accurately recovered the terms. In our experience, linear fits have been adequate to describe the $r$ dependence of the $A_{n}$ and $B_{n}$ values. We have seen significant deviations from this when $N$ is too large, i.e., the fit is including unnecessary higher order terms, providing another indicator for the number of terms required.

6. Remove deformation to circularise the image. The functional forms for $A_{n}(r)$ and $B_{n}(r)$ (step 5) provide the means to repair the deformation. A point $(x, y)$ on the image is shifted to a new value determined by its $(r, \theta)$ position by calculating the shift in $r$ determined by the formula (see the expression for $r_{g}$, Eq. (3))

$$
\Delta r(r, \theta)=\sum_{n=1}^{N} A_{n}(r) \sin n \theta+B_{n}(r) \cos n \theta,
$$

where in general we expect $A_{n}(r)=a_{n} r$ and $B_{n}(r)=b_{n} r$. The intensity at $(r, \theta)$ is shifted to $(r+\Delta r, \theta)$ and mapped to the corresponding $(x, y)$ position on the experimental image. In cases where the position of each ion/electron is retained, the repaired image can be constructed by shifting each of the counts to a new position. The repaired, circularised image is shown in Fig. 3(c). Its PC image is shown in Fig. 4(c) for comparison with the same plot for the original undistorted image in Fig. 4(a). Note the straight lines at the correct positions in the repaired image.
The test of the circularisation process is the extent to which the repaired image matches the original, undistorted image. This comparison is shown in Fig. 3(d), which shows a difference image corresponding to the original, undistorted image minus the circularised image. It can be seen that the circularised image is an excellent match to the original image. Importantly, it shows excellent circularity, with the features at the correct radii. There are some minor differences apparent in Fig. 3(d) and these arise from the pixelated nature of the image leading to a slight broadening of the sharp features, whose FWHM is only $c a$. 1-2 pixels.

\section{EXAMPLES}

In this section, we illustrate the use of the circularisation procedure described above in two examples from the Flinders and ANU laboratories. The first involves photodetachment of $\mathrm{O}^{-}$(ANU) in which there are 6 atomic fine structure transitions, all seen in the image at the high resolution involved. This example provides an excellent test of the ability of the circularisation process to retain the inherent experimental resolution when converting from a complete image to a spectrum. The second example involves photoionisation of benzene (Flinders), a modest resolution experiment but one involving a larger deformation and a significant number of bands, which makes it potentially more challenging to remove the distortions.

Both examples introduce the "real world" issue that adjacent spectral features can overlap within the fluctuations in the radius associated with the radial distortion. In such cases, extracting an individual feature from the full image (step 3) can be problematic. One approach is to extract multiple features and fit to as many Gaussians as there are features. An alternative approach is to carefully mask regions of the image so that only a single feature is extracted. We have taken the latter approach.

\section{A. Photodetachment of $\mathrm{O}^{-}$}

The photoelectron VMI spectrometer at ANU operates at high resolution ( $\mu \mathrm{eV}$ to $\mathrm{meV}$ ), making it critical that photoelectron spectra can be extracted utilising the full inherent resolution of the instrument. Fig. 1 shows an image of the electrons produced by photodetachment of $\mathrm{O}^{-}$at $812.51 \mathrm{~nm}$, $\mathrm{O}^{-}\left({ }^{2} P_{3 / 2,1 / 2}\right)+h v \rightarrow \mathrm{O}\left({ }^{3} P_{2,1,0}\right)+e^{-8,17}$ This image illustrates the problem: although the deformations are comparatively small, non-circularity in the image leads to a radial plot (intensity versus $r$ ), and hence spectrum, that is significantly broader than the inherent resolution of the instrument, as seen by comparing Figs. 1(c) and 1(d). The strongest transition in the image (at $r \sim 1020$ pixels) has a FWHM of 23.6 pixels when the full image is considered, while in a $6^{\circ}$ wedge (i.e., too small for deformations to affect the resolution) the same feature is clearly resolved into two bands. The main peak has a FWHM of $7.12 \pm 0.14$ pixels as determined by a fit to the band using a Gaussian function. (The indicated uncertainty is one standard deviation.) The test for the circularisation procedure is how well this inherent resolution is retained using the full image post-circularisation (assuming that the resolution is maintained across the full image). 
Following the procedure outlined in steps 1-3, an initial estimate for the image centre was used to construct a PC image and the 5 strongest features fit to a trigonometric expansion. From the $\sin \theta$ and $\cos \theta$ terms, the error in the centre position was determined (step 4) and steps 2 and 3 were repeated. A third iteration was not required. Terms up to $N=5$ were required in the trigonometric expansion to reproduce the deformation. The corrections were applied to the image as discussed in steps 5 and 6. The $r$ dependence of $A_{n}$ and $B_{n}$ was well described by a linear function. A PC image of the corrected VMI image is compared with that for the original image in Fig. 2. The straight vertical lines are a marked contrast to the "wiggly" lines from the original image.

An important test for the procedure is the width of the spectral features arising when using the full circularised image. The radial plot shows a significant narrowing of the spectral features, with the intense band at $r \sim 1020$ pixels having a FWHM of $7.19 \pm 0.07$ pixels as determined by a Gaussian fit. Within the uncertainty of the fits, this is the same as the FWHM of $7.12 \pm 0.14$ pixels determined for the $6^{\circ}$ wedge from the original image. Fig. 6 illustrates this. This comparison shows that the circularisation procedure has retained the resolution inherent in the image while allowing the complete image to now be used.

The high resolution of the ANU instrument provides an excellent basis for an analysis of the extent to which the $r$ dependence expected in the image is observed. The energies of the atomic fine structure transitions ${ }^{8,17}$ and the laser wavelength are known. The fine structure transitions are expected to follow the relationship $r^{2} \propto h v-E_{\text {Transition, where } v \text { is the }}$ laser frequency and $E_{\text {Transition }}$ is the energy of the fine structure transitions. The electron kinetic energy is thus $E_{\text {Kinetic }}$ $=h v-E_{\text {Transition }}$ and a plot of $r$ versus $E_{\text {Kinetic }}^{1 / 2}$ should be linear with an intercept of zero. It is found that the expected relationship holds quite closely, but slightly better agreement is given for a quadratic form $r=a E_{\text {Kinetic }}^{1 / 2}+b E_{\text {Kinetic. }}$. A plot of the residuals (observed-fit) for the linear and quadratic fits is

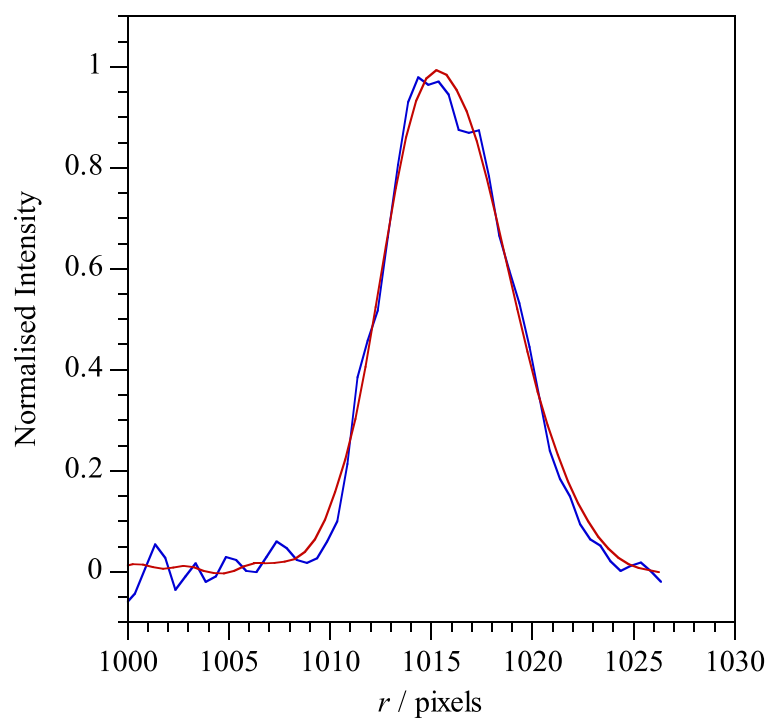

FIG. 6. A plot of the ${ }^{3} P_{2} \leftarrow{ }^{2} P_{3 / 2}$ transition seen in photodetachment of $\mathrm{O}^{-}$near 1015 pixels showing how well the full circularised (red curve) image line width matches that for the $6^{\circ}$ wedge in the uncircularised image (blue curve). This is the most intense feature in the image.

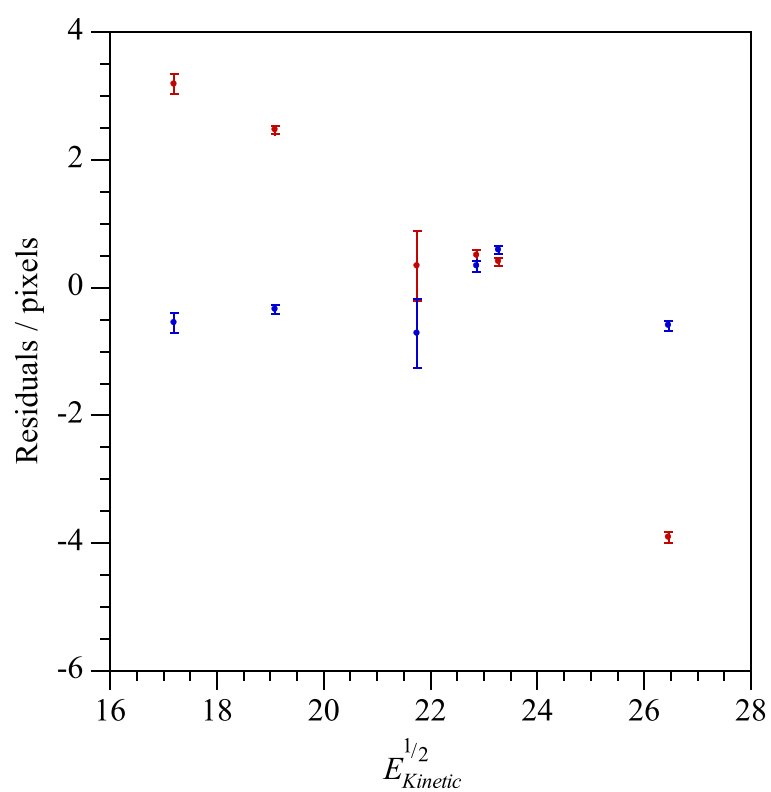

FIG. 7. The residuals from linear (red) and quadratic (blue) fits of a plot of

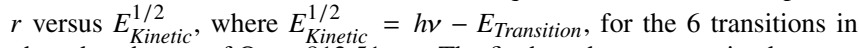
photodetachment of $\mathrm{O}^{-}$at $812.51 \mathrm{~nm}$. The fits have been constrained to pass through $(0,0)$. The error bars correspond to three standard deviations from Gaussian fits to each transition.

shown in Fig. 7. This is an example of a very small $r$ dependent deformation remaining in the image post-circularisation. Since the functional form for the radial dependence has been determined, the image could be further processed to remove the non-linearity. The complete "repair function" in this case involves circularisation followed by linearisation.

\section{B. $1+1$ photoionisation of benzene via 60}

VMI images of photoelectrons produced by $1+1$ resonance enhanced multiphoton ionisation (REMPI) of benzene via vibrational states associated with the $\mathrm{S}_{1}\left({ }^{1} \mathrm{~B}_{2 \mathrm{u}}\right) \leftarrow \mathrm{S}_{0}\left({ }^{1} \mathrm{~A}_{1 \mathrm{~g}}\right)$ transition have been reported by a number of authors. ${ }^{26-28}$ The vibrational level structure in the benzene cation leads to a rich set of closely spaced features in the photoelectron images. The effects of deformations in the image are acute in such cases due to the potential merging of features in extracted spectra.

A VMI image of the photoelectrons produced following REMPI via the $6_{0}^{1}$ transition, measured in the Flinders laboratory, is shown in Fig. 8(a). While not obvious to the eye, the image is distorted into a slanted oval shape (for example, for the outermost ring (electron energy $2659 \mathrm{~cm}^{-1}$ ) the short radius is 2 pixels smaller than the large radius) and the upper right hand quadrant is slightly compressed. The deformation is clear in the PC image, Fig. 8(b). The deformation is larger than that found for the $\mathrm{O}^{-}$image: based on the trigonometric function required to repair this image, the distortion is a factor of $\sim 4.5 \times$ larger than was seen in the $\mathrm{O}^{-}$image discussed in Section III A. The benzene image also has fewer counts, and the larger deformation coupled with the reduced signal makes this a more challenging example of the circularisation procedure. Since the issue of resolution in circularised images has been addressed in detail in the high resolution example above, the focus in this example is on the straightening of the lines in the PC image. 
(a)

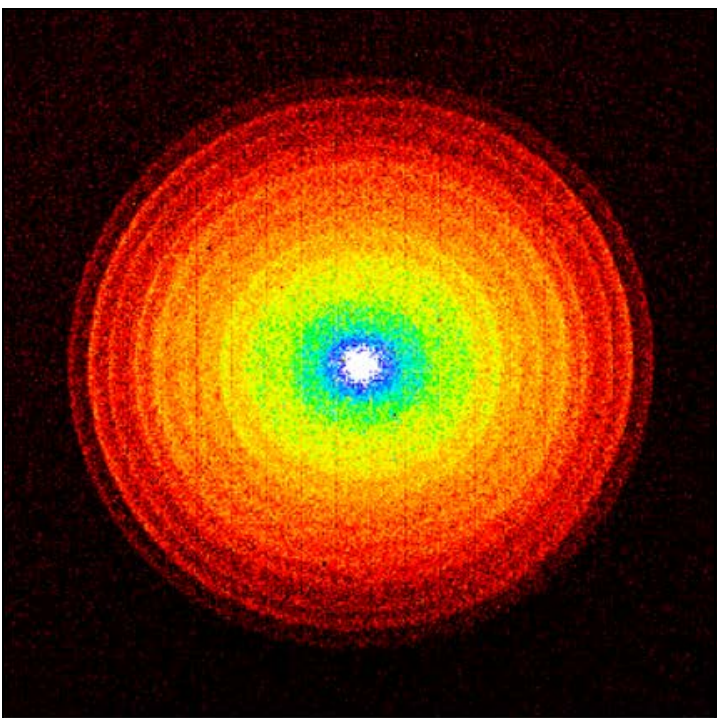

(b)

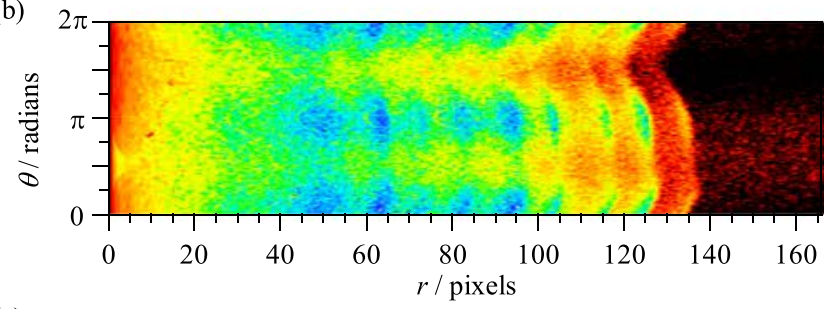

(c)

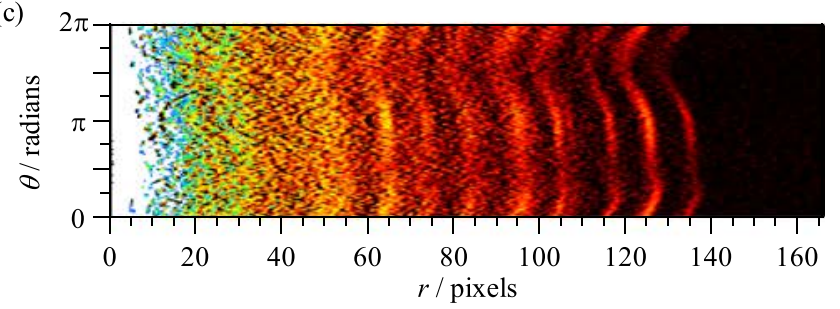

(d)
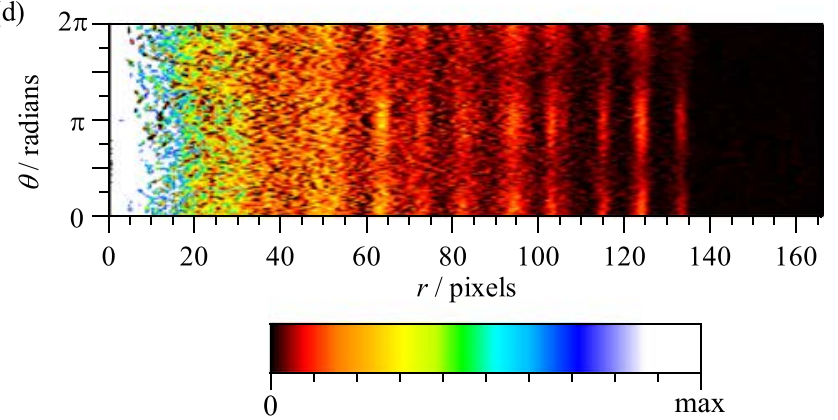

FIG. 8. (a) A VMI image of the photoelectrons produced following REMPI of benzene via the $6_{0}^{1}$ transition measured in the Flinders laboratory. (b) A polar coordinate (PC) image of the VMI image shown in (a). Note how the features merge and blur into each other in this image. (c) The inverse Abel transformed image of (b), which allows the features to be readily identified. The intensity in the PC image was divided by $r$ prior to applying the inverse Abel transformation (see text). (d) The corresponding image (c) for the circularised VMI image showing that the wiggly vertical lines have been straightened by the circularisation process.

An initial estimate for the image centre was used to construct a PC image and the 7 strongest features fit to a trigonometric expansion (steps 1-3). It can be seen from Fig. 8(b) that finding the peak positions at each angle is not straightforward in this example because with many spheres being compressed onto the detector there is a buildup of counts as $r$ decreases. The advantage afforded by taking the inverse Abel transformation of this image to identify peak positions is apparent by comparing Figs. 8(b) and 8(c). As expected, the initial centre required correction (step 4), as determined from the $\sin \theta$ and $\cos \theta$ terms, necessitating a second iteration of steps 2 and 3. As was found for the previous two cases, a third iteration was not required as the shift indicated by the $\sin \theta$ and $\cos \theta$ terms was zero within the uncertainty in the intercepts. The inverse Abel PC image for the uncorrected VMI image is shown in Fig. 8(c). It was found that terms up to $N=3$ in the trigonometric expansion were sufficient to fit the deformation. Applying the corrections to the image (steps 5 and 6) yielded the inverse Abel PC image shown in Fig. 8(d). Again, a linear function was sufficient to describe the $r$ dependence of $A_{n}$ and $B_{n}$. Comparison of Figs. 8(c) and 8(d) shows that the circularisation process has straightened the features in the PC plot in this more difficult example.

\section{REPAIR BY CALIBRATION}

The process described in Section II and illustrated with experimental examples in Section III involves repairing distorted images using the image itself. The "distortion repair function" determined enables the intensity at a position $(x, y)$ on the image to be reassigned to the position $\left(x^{\prime}, y^{\prime}\right)$ based on the $(r, \theta)$ coordinates for $(x, y)$. In principle, this correction function need not be determined using the distorted image to be corrected but could be pre- or post-determined from a calibration experiment that provides appropriate coverage across the image. This is discussed in the Appendix.

\section{CONCLUSIONS}

A method to repair distortions in VMI images has been presented based on fitting the angular behaviour of each of the ring structures in the image to a trigonometric expansion. The resulting function allows the intensity at any value of radius and angle to be mapped to a new position that removes the distortion and returns the features to circular. The method has been successfully applied to repair distorted images in two photoelectron spectra. It has been demonstrated that the resolution achieved using the whole of the repaired image matches that of a small wedge in the original image, showing that the technique allows the complete image to be processed at the resolution inherent to the instrument. While the method relies on the analysis of the structure in an image, it could also be applied to determine the "repair function" using a calibration image (or series of images) prior to or post the experiment. One outcome of the analysis is that it enables the image centre to be determined with high accuracy. Once the image has been circularised it can be processed by any of the approaches that have been developed for that purpose.

The fitting method utilises an inverse Abel transformation of the image in polar coordinates as a means to reshape the image into a series of spectral features in order to determine the radial position of features at each angle. Although the velocity distribution is not in general spherically symmetric and so this is not a mathematically correct means to 
extract the velocity distribution, the feature positions are accurately reproduced in the resulting spectrum while the intensity and anisotropy parameters can be remarkably close to those obtained using the proper inverse Abel transformation of the image.

The computer code developed for the circularisation process is available for use by the VMI community. This code includes the ability to perform the inverse Abel analysis of images. Reference 29 provides the URL and DOI for accessing this material and the instructions for using it.

\section{SUPPLEMENTARY MATERIAL}

See supplementary material for a description of (i) the basis for a trigonometric fit to polar coordinate plots to extract the centre of VMI images and (ii) a comparison of the inverse Abel transformation of velocity map images in Cartesian and polar coordinates.

\section{ACKNOWLEDGMENTS}

J.R.G. and W.D.L. wish to acknowledge the staff of the Electronics and Mechanical Workshops who contributed significantly to the construction and maintenance of the Flinders VMI apparatus. The $\mathrm{O}^{-}$photoelectron spectrum of Fig. 1 was measured as part of the electron anisotropy study of Dr. S. J. Cavanagh. ${ }^{17}$ S.T.G. acknowledges support by the Australian Research Council Discovery Project Grant No. DP160102585.

\section{APPENDIX: REPAIR BY PRE-CALIBRATION}

The repair process described can use a calibration species that provides appropriate coverage across the image to determine the "distortion repair function." Since the distortions are typically generated by stray fields, they depend on the mass of the species detected and so it is preferable in the case of ions to match the mass of the species used for the calibration to that used in the experiments as closely as possible.

In a calibration experiment, the correction would be generated from multiple images of a particular feature, or features, of known energy. By varying the translational energy associated with the feature(s), which is straightforwardly achieved by using different wavelengths in the dissociation/ionising/photodetaching laser, one generates a set of images consisting of one or more (distorted) rings of known translational energy, $E$. The radius $r$ of the ring is proportional to $E^{1 / 2}$ in the absence of distortion and so to obtain calibration rings separated by an approximately constant change in radius, $\Delta r$, the calibration images should be taken using wavelengths that generate constant, or near constant, steps in $E^{1 / 2}$. Since the detector must be calibrated to determine the specific relationship between radius and energy, acquiring the additional calibration images for removing distortion is not a significant experimental overhead, although it does require that the calibration be performed using a calibrant of appropriate mass.
In this application, the calibration images would be analysed in the manner described in Section II including, if required, a final radial linearisation as discussed in Section III A. The correction function obtained is used to correct the images measured in the proper experiment. In this case, the measured event positions could also be assigned their repaired position as they are recorded and the experimental image built up with the deformations removed. Such a procedure requires that the experimental apparatus is stable and reproducible during the calibration and experimental data collection.

${ }^{1}$ D. W. Chandler and P. L. Houston, J. Chem. Phys. 87, 1445 (1987).

${ }^{2}$ A. T. J. B. Eppink and D. H. Parker, Rev. Sci. Instrum. 68, 3477 (1997).

${ }^{3}$ Imaging in Molecular Dynamics: Technology and Applications, edited by B. J. Whitaker (Cambridge University Press, Cambridge, 2003).

${ }^{4}$ Imaging in Chemical Dynamics: The State of the Art, edited by A. G. Suits and R. E. Continetti (ACS Symposium Series, 2000), Vol. 770.

${ }^{5}$ A. G. Suits, M. Kawasaki, and W. D. Lawrance, Phys. Chem. Chem. Phys. 8, 2913-2914 (2006).

${ }^{6}$ A. Osterwalder, M. J. Nee, J. Zhou, and D. M. Neumark, J. Chem. Phys. 121, 6317 (2004).

${ }^{7}$ G. J. Rathbone, T. Sanford, D. Andrews, and W. C. Lineberger, Chem. Phys. Lett. 401, 570 (2005).

${ }^{8}$ S. J. Cavanagh, S. T. Gibson, M. N. Gale, C. J. Dedman, E. H. Roberts, and B. R. Lewis, Phys. Rev. A 76, 052708 (2007).

${ }^{9}$ A. T. J. B. Eppink, S.-M. Wu, and B. J. Whitaker, in Imaging in Molecular Dynamics: Technology and Applications (Cambridge University Press, Cambridge, 2003), Chap. 3, pp. 65-112.

${ }^{10}$ F. Renth, J. Riedel, and F. Temps, Rev. Sci. Instrum. 77, 033103 (2006).

${ }^{11}$ B. Dick, Phys. Chem. Chem. Phys. 16, 570 (2014).

${ }^{12}$ C. R. Gebhardt, T. P. Rakitzis, P. C. Samartzis, V. Ladopoulos, and T. N. Kitsopoulos, Rev. Sci. Instrum. 72, 3848 (2001).

${ }^{13}$ D. Townsend, M. P. Minitti, and A. G. Suits, Rev. Sci. Instrum. 74, 2530 (2003).

${ }^{14}$ E. W. Hansen and P.-L. Law, J. Opt. Soc. Am. A 2, 510 (1985).

${ }^{15}$ J. R. Gascooke, Ph.D. thesis The Flinders University of South Australia, 2000.

${ }^{16}$ M. Ryazanov, Ph.D. thesis University of Southern California, 2012.

${ }^{17}$ S. J. Cavanagh, S. T. Gibson, and B. R. Lewis, J. Phys.: Conf. Ser. 212, 012034 (2010).

${ }^{18}$ S. J. Cavanagh, S. T. Gibson, and B. R. Lewis, J. Chem. Phys. 137, 144304 (2012).

${ }^{19}$ B. R. Visser, M. A. Addicoat, J. R. Gascooke, W. D. Lawrance, and G. F. Metha, J. Chem. Phys. 138, 174310 (2013).

${ }^{20}$ B. R. Visser, M. A. Addicoat, J. R. Gascooke, X. Zhang, K. Bowen, W. D. Lawrance, and G. F. Metha, Chem. Phys. Lett. 625, 164 (2015).

${ }^{21}$ B. R. Visser, M. A. Addicoat, J. R. Gascooke, W. D. Lawrance, and G. F. Metha, J. Chem. Phys. 145, 044320 (2016).

${ }^{22}$ S. Manzhos and H.-P. Loock, Comput. Phys. Commun. 154, 76 (2003).

${ }^{23}$ V. Dribinski, A. Ossadtchi, V. A. Mandelshtam, and H. Reisler, Rev. Sci. Instrum. 73, 2634 (2002).

${ }^{24}$ The PyAbel suite provides functions for forward and inverse Abel Transform and several methods for determining the image centre, including identifying the center of mass, by convolution of two projections along each axis, and by breaking the image into slices, and comparing them for symmetry: see doi: 10.5281 /zenodo.47423.

${ }^{25}$ K. L. Reid, Annu. Rev. Phys. Chem. 54, 397 (2003).

${ }^{26}$ Y. Ogi, H. Kohguchi, D. Niu, K. Ohshimo, and T. Suzuki, J. Phys. Chem. A 113, 14536 (2009).

${ }^{27}$ D. M. Niu, Y. Ogi, Y. I. Suzuki, and T. Suzuki, J. Phys. Chem. A 115, 2096 (2011).

${ }^{28}$ Z. H. Qu, C. S. Li, Z. B. Qin, X. F. Zheng, G. X. Yao, X. Y. Zhang, and Z. F. Cui, Rev. Sci. Instrum. 86, 063106 (2015).

${ }^{29}$ J. R. Gascooke and W. D. Lawrance, "FITS viewer and VMI analysis: A program for analysing and circularising VMI images," see doi: $10.5072 / 86 / 58 d d d 44294742$. 